\title{
NEW MODELING TECHNIQUE FOR BIONIC SPACE GRID STRUCTURES
}

\author{
N. Li and Y.Z. Luo* \\ Space Structures Research Center, Zhejiang University, Hangzhou, China \\ *(Corresponding author: E-mail: luoyz@zju.edu.cn)
}

Received: 31 October 2007; Revised: 15 December 2007; Accepted: 20 December 2007

\begin{abstract}
This paper is intended to introduce a new modeling technique for bionic space grid structures. Utilizing the methods derived from bionics and reverse engineering, the modeling technique acquires geometric data points of the natural patterns. Generally, the theory of bionics is used to express the forms of spatial patterns in direct analytic function of curves or surfaces. In this paper, the key point is to express obvious geometric features in data points. For more complicated natural patterns, it is convenient to get data points by three-dimensional (3D) measurement in reverse engineering. So this paper introduces the 3D measurement and explains the manipulations of the data points detailedly. Then the free-form surface of the natural pattern is reconstructed and meshed by the re-interpolation reflection meshing method, which combines the bi-cubic B-spline interpolation of surface and reflection meshing method. In order to satisfy the practical requirement, several meshing ways and evaluation functions of grid quality are proposed. There are several illustrated design examples are presented.
\end{abstract}

Keywords: Modeling technique; space grid structure; bionics; reverse engineering; B-splines interpolation; meshing

\section{INTRODUCTION}

Generally speaking, the forms of space grid structures are usually expressed in regular shape. Take the space truss or latticed shell for example. Its form is easily designed in a plane, a semi-cylinder, a semi-sphere and so on. But nowadays, there are some special bionic space grid structures, which imitate the natural patterns vividly, appearing in public buildings. Since their elements can neatly express the free-form surface, their forms become diversified in the development. Such as the aim in the "Bubble" (Figure 1), the given name for the Exhibition-Pavillon of BMW at the International Motorshow IAA in Frankfurt in 1999, was to support the formal idea by expressing the dynamic balance of the two water drops becoming a unit and suggesting the idea that form is only a "frozen moment" (Harald [1]).

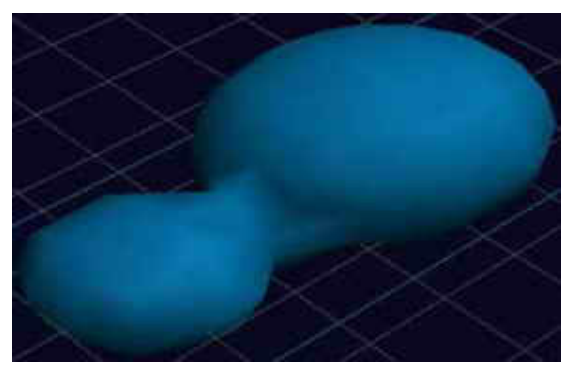

(a) Form Simulation

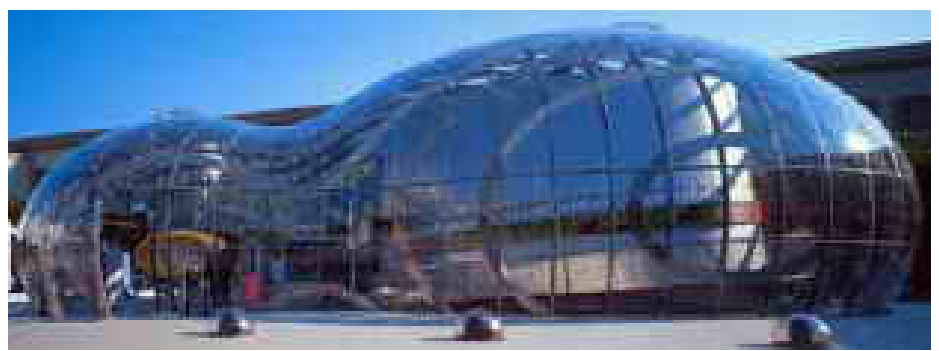

(b) Final Project

Figure 1. Bubble-Project 
However, it is quite difficult to rebuild the modeling of the natural pattern, as this free-form surface can not be represented by the direct analytic function. For this reason it is easy to understand the interested designers have different techniques like bionics and mathematics theory (Stach [2]). The data points of the surface can be acquired by methods derived from bionics or reverse engineering, and then can be used to reconstruct the whole surface by techniques of mathematics. So the modeling technique applied in this paper begins with the acquisition of the data points, and its essential process is to rebuild the space grid structure from the data points.

At present, there are two methods to get the data points. The one is basic geometric feature extraction and the other is three-dimensional (3D) measurement. The basic geometric feature extraction comes from the theory of bionics. Raup [3] found that the surface of seashells could be expressed by four basic parameters. Stach [2] described three familiar seashells with those basic parameters and obtained the corresponding analytical curves. The 3D measurement is derived from the reverse engineering of geometric models. Reverse engineering typically starts with measuring an existing object so that a surface or solid model can be deduced in order to exploit the advantages of CAD/CAM technologies. The 3D measurement comprises a lot of methods, such as optical, acoustic, magnetic methods and so on (Várady et al. [4]). In all of them, the laser scanning is used most commonly, which is an optical non-contact method and uses 3D scanner to get points. Barber et al. [5] concluded that the data acquisition had greatly progressed in these last years due to the introduction of laser scanning technologies. Balz and Böhm [6] outlined the point clouds of the morphology scanned by 3D scanner. According to Harald [1], in the design process of M.art.A. Museum in Herford, a series of physical models were built manually by architects and then were three-dimensionally digitized by using 3D scanner. The resulted data were transformed in the computer-aided design environment to correct the shape and define the digital database.

After the acquisition of data points, some techniques of mathematics can be applied to rebuild the practical structures. Gong [7] first introduced the theory of computer-aided geometric design (CAGD) into the modeling technique of space grid structure. Then the reflection meshing method was applied to deal with the free-form surface, and evaluation functions of grid quality were proposed (Zhang [8]). Cen [9] advanced the re-interpolation reflection meshing method and completed the program to realize a variety of shells. There were several practical meshing ways extended to divide the surface ( $\mathrm{Li}$ and Luo [10]). The modeling technique combines these methods, and furthermore, in order to deal with the problem of data points which are not of rectangular topology, the cubic B-spline interpolation of curve will be used.

According to the above introduction, the modeling technique in this paper comprises two important phases. The first one is data acquisition, and the second one is surface reconstruction and meshing. Especially in the first phase, the modeling technique utilizes both geometric feature extraction and 3D measurement to get data points. Generally, the theory of bionics is used to express the forms of spatial patterns in direct analytic function of curves or surfaces. In this paper, the geometric feature extraction derived from bionics expresses the obvious geometric features in data points. For more complicated natural patterns, the 3D measurement is used to get data points conveniently. So this paper introduces this method and explains the manipulations of the data points detailedly. The second phase of surface reconstruction and meshing which have been improved continuously is also described to complete the whole modeling. Several design examples will be presented to illustrate the technique. 


\section{DATA ACQUISITION}

This section will expatiate on the two methods, basic geometric feature extraction and 3D measurement, which get the data points from the surface of natural pattern rationally.

\subsection{Basic Geometric Feature Extraction}

Through observing the scallop, pilosa and other spatial patterns, it is found that the geometric features can be expressed by base-lines and ridge-lines, as shown in Figure 2. The base-lines determine the basic shape of the pattern while the ridge-lines determine the curvature.
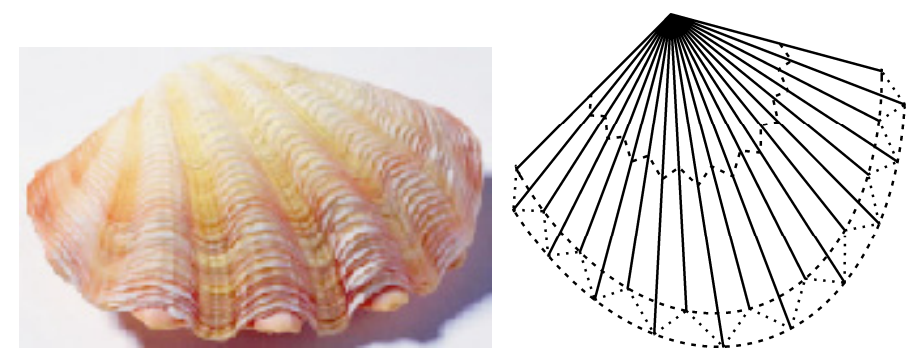

(a) Scallop
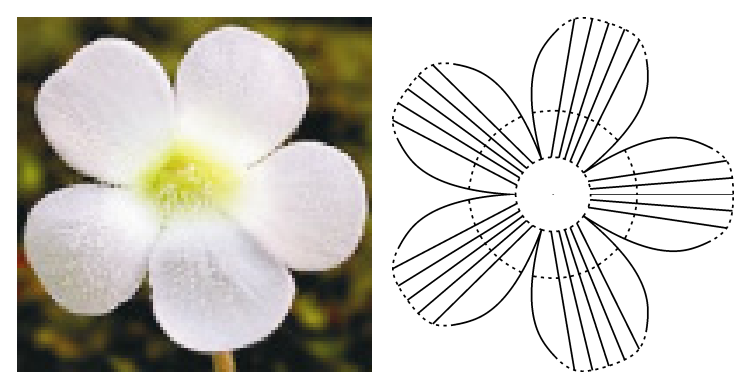

(b) Pilosa

Figure 2. Natural Patterns

The chain Dots Denote the Base-lines, and the Solid Lines Denote the Ridge-lines.

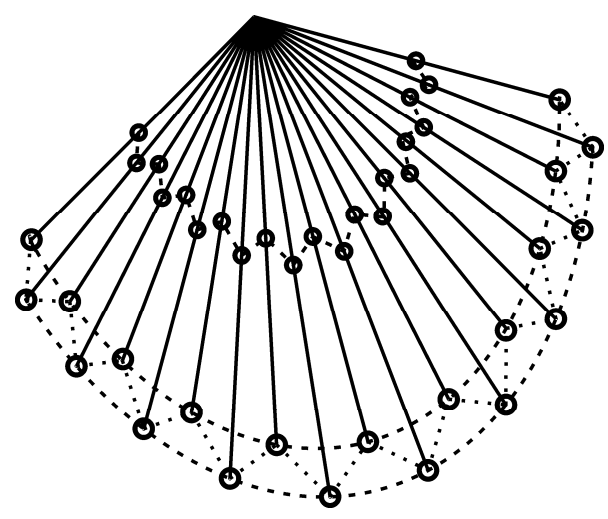

Figure 3. Data Points

The Open Circles Express the Position of Data Points. 
Data points are extracted from the base-lines and ridge-lines. Figure 3 shows that the data points are of topological relationship and consist of intersection.

To get the ideal curvature, the data points, which are placed on middle of ridge-lines, can be adjusted in two ways, which are named z-coordinate offset and expansion coefficient set respectively, as shown in Figure 4. In Cartesian coordinates, z-coordinate offset modifies $\mathrm{z}$ coordinate of the data points to target position, and the expansion coefficient set changes the size of the adjustable ring, which consists of data points of the same z-coordinate.

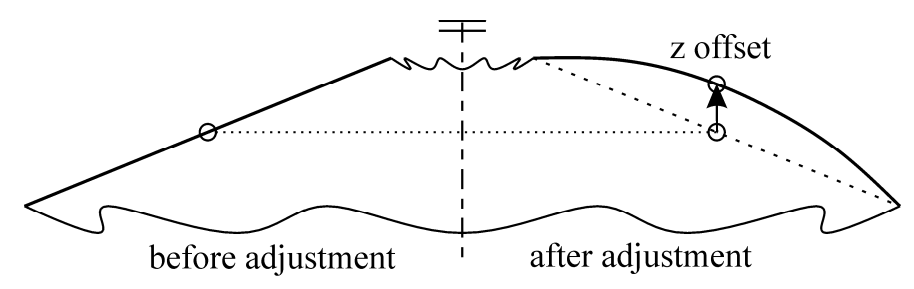

(a) Z-Coordinate Offset

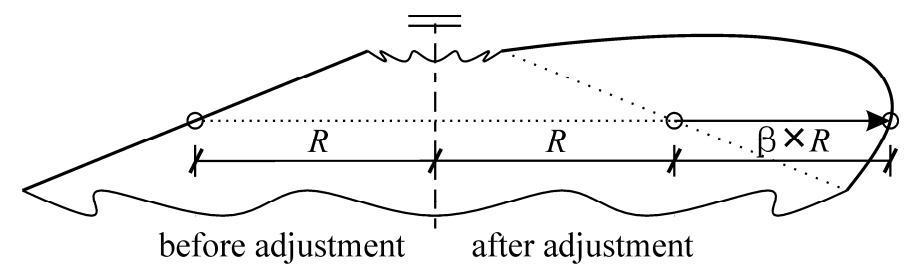

(b) Expansion Coefficient Set. $\beta$ is the Expansion Coefficient.

Figure 4. The Ways of Adjustment

\subsection{D Measurement}

With the rapid development of 3D measurement technology, it becomes easier to get precise data points from the natural patterns. In this paper, the important point is to explain the manipulations of the data points, so it will not refer to the instruction for the equipment of 3D measurement.

\subsubsection{Point clouds}

By 3D measurement, a large amount point data of physical surface can be recorded, which are 3D coordinates and stored in computer with certain files. These point data are so-called point clouds. For example, the advantages of the laser scanning are its capacity to store large 3D point clouds acquired with a high speed and a high level of precision (Luca et al. [11]). In Figure 5a and Figure $6 \mathrm{a}$, the point clouds show cylindrical and conical surfaces. In Figure $7 \mathrm{a}$, the point clouds come from a dolphin model, so its surface is free-form. All of these point clouds are scattered.

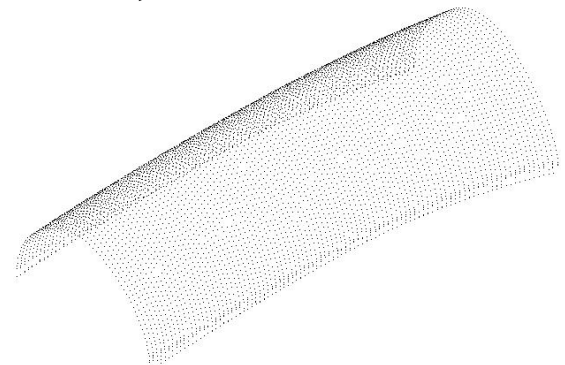

(a) Point Clouds

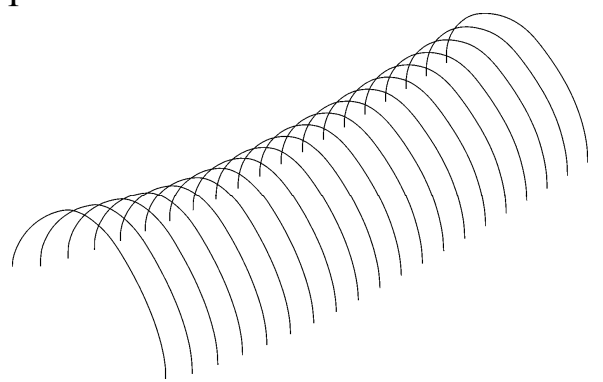

(b) Data Points

Figure 5. Cylindrical Surface 


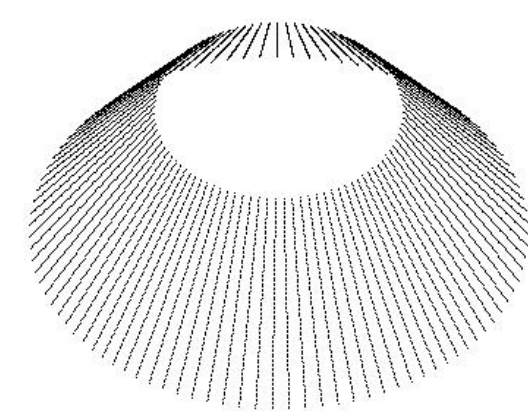

(a) Point Clouds

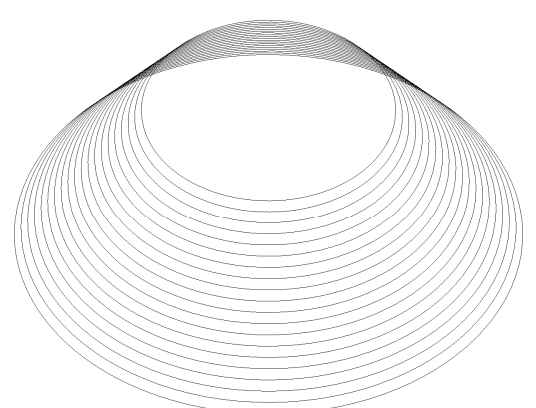

(b) Data Points

Figure 6. Conical Surface

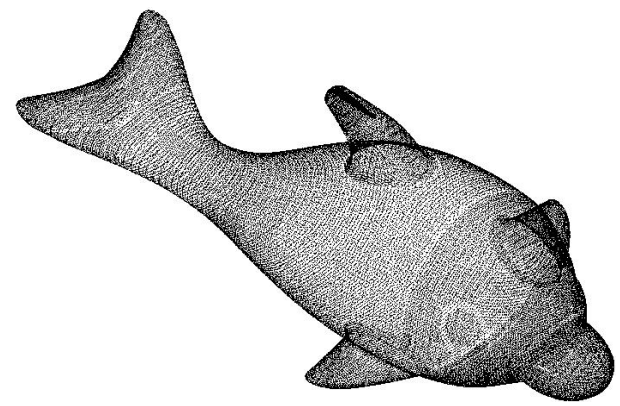

(a) Point Clouds

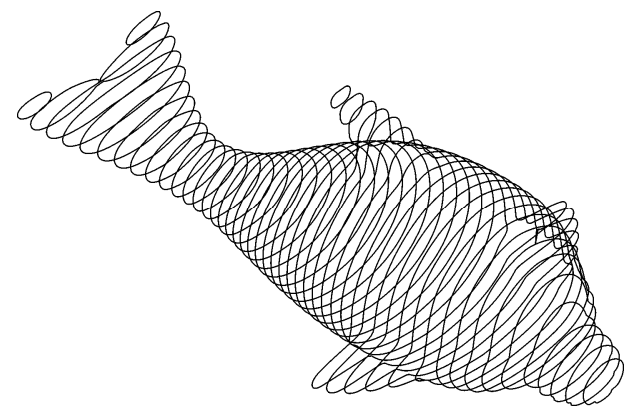

(b) Data Points

Figure 7. Dolphin-Shaped Surface

\subsubsection{Data points extraction}

After the point clouds have already acquired, the results are transformed in the CAD environment of Imageware to delete the noise, subtract the redundancy and so on. Then the data points can be extracted from the sections of point clouds.

In reverse engineering, surface reconstruction is the most important process. But in this modeling technique, the main purpose is to rebuild the space grid structures. In order to get ready for the surface reconstruction and meshing process, the data points should distribute in rectangular topological relationship. But as shown in Figure 5b, each solid line may have different number of data points, and the data points may still distribute scattered. It is necessary to pretreat these data points before the surface reconstruction and meshing process.

\section{SURFACE RECONSTRUCTION AND MESHING METHOD}

In this section, the cubic B-spline curve will be used to deal with the problem of data points which are not of rectangular topology. Then the re-interpolation reflection meshing method will be used to rebuild the space grid structures. Before the explanation of the procedure, the theory of B-splines will be introduced briefly.

\subsection{B-Spline Representation}

The definition, important properties and manipulations of B-spline curve and surface are briefly outlined here. The detailed contents can be found in Farin [12] and DeBoor [13]. 


\subsubsection{B-splines interpolation of curve and surface}

A B-spline curve is defined by the Eq. 1.

$$
\boldsymbol{p}(u)=\sum_{i=0}^{n} \boldsymbol{d}_{i} N_{i, k}(u) \quad 0 \leq u \leq 1,
$$

where $N_{i, k}(u)$ are B-spline basis functions, $\boldsymbol{d}_{i}$ are vectors composed of $\mathrm{x}, \mathrm{y}, \mathrm{z}$ coordinates of the control points, $k$ is the order of a B-spline curve, and $\boldsymbol{U}=\left[u_{0}, u_{1}, \ldots, u_{m}\right]$ is the knot vector which specifies the distribution of the parameter $u$ along the curve. The relationship among the order of the curve $k$, the number of control points $n+1$, and the number of knots $m+1$ is given by

$m+1=n+1+k+1$.

Figure 8 depicts the non-uniform cubic B-spline curve interpolated by the data points.

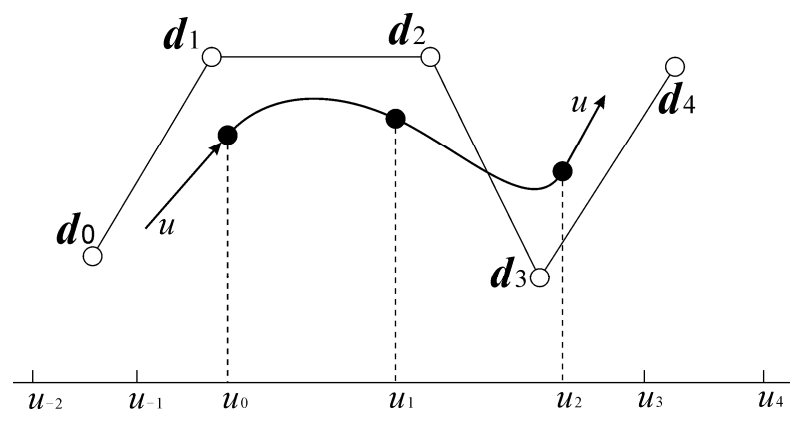

Figure 8. Non-Uniform Cubic B-Spline Curve Fitting

B-spline basis functions $N_{i, k}(u)$ are defined by

$$
\begin{aligned}
& N_{i, 0}(u)= \begin{cases}1, & \text { if } u_{i} \leq u<u_{i+1} \\
0, & \text { otherwise }\end{cases} \\
& N_{i, k}(u)=\frac{u-u_{i}}{u_{i+k}-u_{i}} N_{i, k-1}(u)+\frac{u_{i+k+1}-u}{u_{i+k+1}-u_{i+1}} N_{i+1, k-1}(u),
\end{aligned}
$$

where $i$ is sequence number, $k$ is the order. These recursive formula show that it needs $k+2$ knots $u_{i}$, $u_{i+1}, \ldots, u_{i+k+1}$ to calculate $N_{i, k}(u)$, and the interval $\left[u_{i}, u_{i+k+1}\right]$ is called the support knot span of $N_{i, k}(u)$.

As an extension of a parametric B-spline curve, a biparametric B-spline surface can be defined as the tensor product of B-spline curves. A $(k \times l)$ th order B-spline surface is defined as follows:

$$
\boldsymbol{p}(u, v)=\sum_{i=0}^{m} \sum_{j=0}^{n} \boldsymbol{d}_{i, j} N_{i, \mathrm{k}}(u) N_{j, 1}(v), \quad 0 \leq u, v \leq 1
$$

where $(m+1) \times(n+1)$ control points $\boldsymbol{d}_{i, j}$ build up the control net in $3 \mathrm{D}$, and $N_{i, k}(u), N_{j, l}(v)$ are the $\mathrm{B}$-spline basis functions of order $k$ and $l$ in the $u$ - and $v$-directions, respectively. Figure 9 shows various geometric entities defining a bi-cubic non-uniform B-spline patch. 


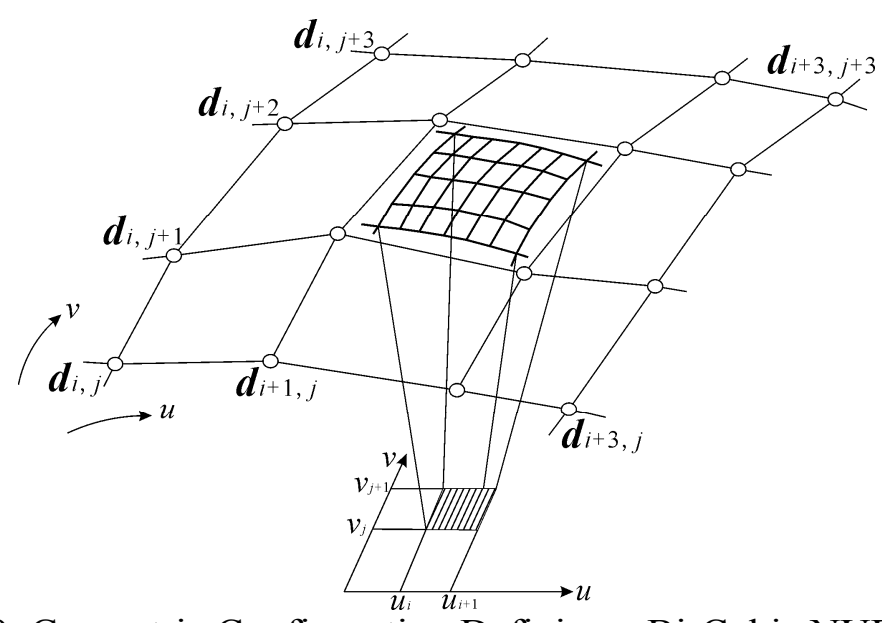

Figure 9. Geometric Configuration Defining a Bi-Cubic NUBS Patch

\subsubsection{Evaluation of B-spline surface}

The overall procedure of constructing a composite B-spline surface from the input data consists of the following four separate steps:

(1) Determination of knot spans $U=\left[u_{0}, u_{1}, \ldots, u_{m}\right]$ and $V=\left[v_{0}, v_{1}, \ldots, v_{n}\right]$.

The spline interpolation problem is usually stated as "given data points $x_{i}$ and parameter values $u_{i} . .$. . In practice, parameter values are rarely given and therefore must be made up somehow. The convenient way to determine the $u_{i}$ is to have the knot spacing proportional to the distances of the data points.

$\frac{u_{i}-u_{i-1}}{u_{i+1}-u_{i}}=\frac{\left\|x_{i}-x_{i-1}\right\|}{\left\|x_{i+1}-x_{i}\right\|}$.

A knot sequence satisfying Eq. 6 is called chord length parametrization. In the present study, this method is employed.

(2) Determination of intermediate control points.

At this step, a B-spline curve is fitted from each "column" $j$ of the input data. That is, for each column $j$, sequence mesh points and end boundary tangents are given. The intermediate control points corresponding to the input data are found.

(3) Determination of boundary vectors.

The next step is to determine the boundary vectors from the boundary tangents and corner twist vectors. The boundary tangents can be determined by using data points.

(4) Determination of control vertices $\boldsymbol{d}_{i, j}$.

This step determines the B-spline control points $\boldsymbol{d}_{i, j}$ by fitting a composite B-spline curve from each "row" $i$ of intermediate control points.

In the present study, the B-spline curve or surface is cubic, so the order $k$ or $l$ is three.

\subsection{Data Points Manipulations}

The B-spline interpolation of surface request data points distribute in rectangular topological relationship. But in the manner of $3 \mathrm{D}$ measurement, some data points may not fulfil this 
requirement. As shown in Figure 10a, the data points distribute in lines, so the problem can be solved with cubic B-spline curve. Through the interpolation, it ensures the number of data points in each line is equal and the data points distribute in rectangular topological relationship. The data points which have been manipulated can be seen in Figure 10b.

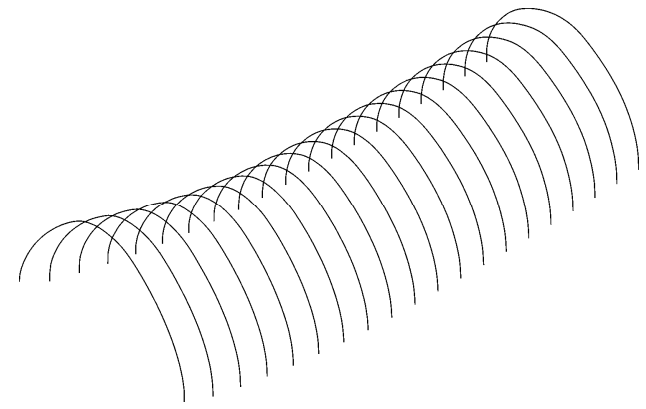

(a) Before Interpolation of B-Spline Curve

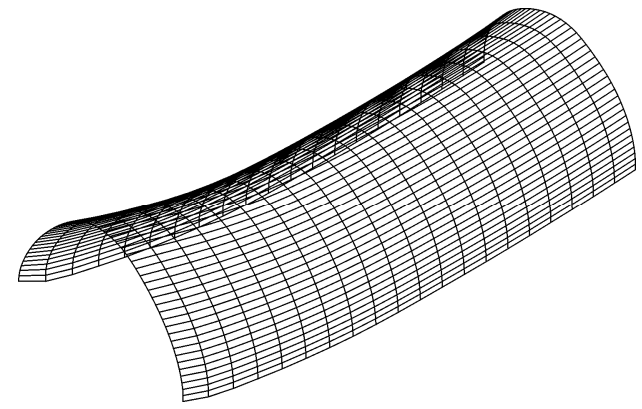

(b) After Interpolation of B-Spline Curve

Figure 10. Data Points Manipulation

\subsection{Re-Interpolation Reflection Meshing Method}

A rectangular parameter field has been prepared for the B-spline interpolation of surface, and it is also appropriate for the reflection meshing method to divide the surface. The basic procedure of the reflection meshing method is as follows (Zheng, et al. [14]):

Mesh the rectangular parameter field and get knots $\left(u_{i}, v_{j}\right)$.

(2) Substituting each knot into Eq. 5, gives the corresponding $p\left(u_{i}, v_{j}\right)$.

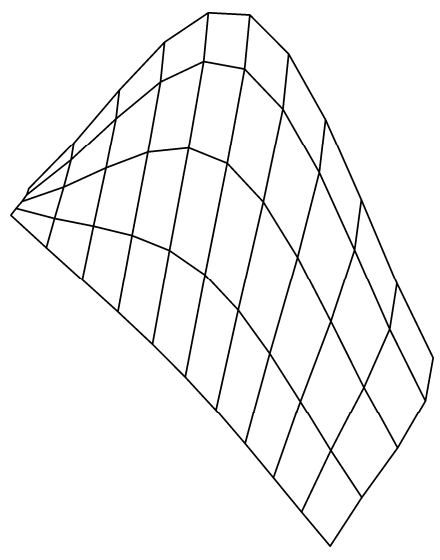

(a) Single Interpolation

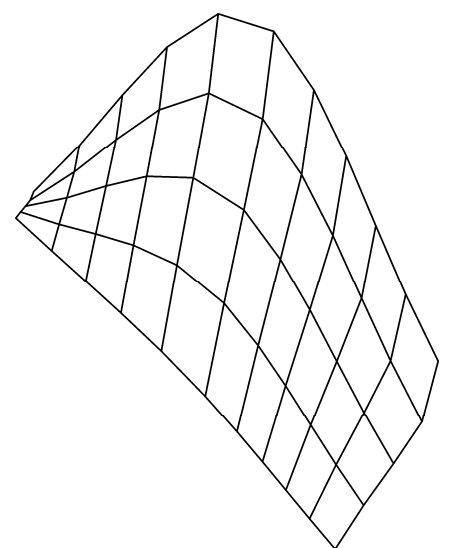

(b) Re-Interpolation

Figure 11. Comparison of Meshing Methods

Reflection meshing method is very simple, and if the parameter values are given well, it will produce a good division. However, in the process of B-Spline interpolation, the data points are parameterized with chord length parametrization, which is an approximate method compared with the arc length parametrization. If the curvature changes sharp, the parameter values will become anamorphic, and the inherent flaw is that some uneven grids (Figure 11a) will appear in the places where the curvature changes sharp. 
Therefore, the re-interpolation reflection meshing method is proposed to advance the prior method. The procedure is as follows:

(1) From the data points, the bi-cubic B-spline interpolation of surface and reflection meshing method are applied to get the output of enough new data points, which are rectangular topology and express the features of the surface clearly.

(2) Put the new data points repeat the above procedure, and the required grids will be acquired.

This approach is similar to the arc length parametrization, and the final grids distribute regular and even. As shown in Figure 11b, grids tend to be more homogeneous.

\subsection{Meshing Ways}

To facilitate modeling, meshing ways can be designed as the following solutions (Li and Luo [10]):

(1) Rectangular grids:

- Set the number of the round and radial grids. This solution is suitable when the number of grids has been determined.

- Set the expectation of the element length, then the surface can be meshed automatically. It is suitable when the length of the element has been restricted.

- In addition, the grids can be decreased rationally to avoid too many grids centralizing on the top.

(2) Three-dimensional grids:

- Set the number of central round and radial grids.

Take the conical surface for example. The surfaces which are meshed by the above solutions are shown in Figure 12.

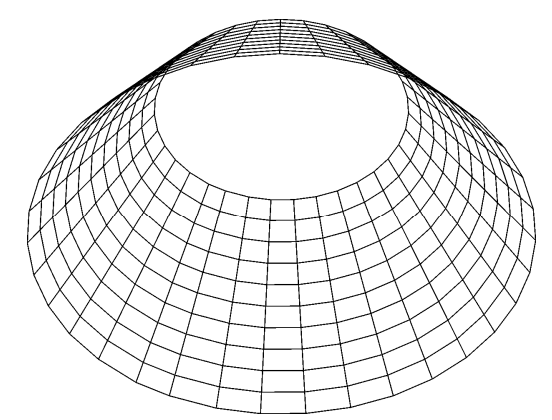

(a) Rectangular Grids: Set Number

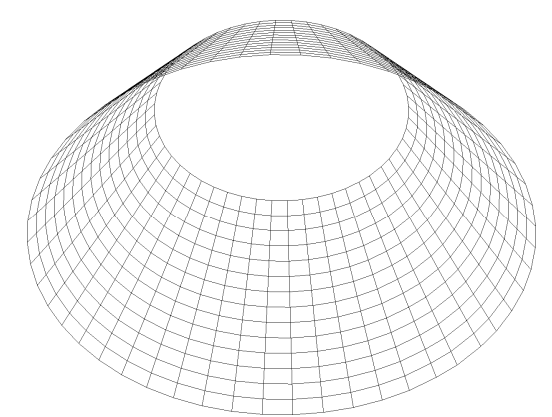

(b) Rectangular Grids: Set Expectation of The Element Length 


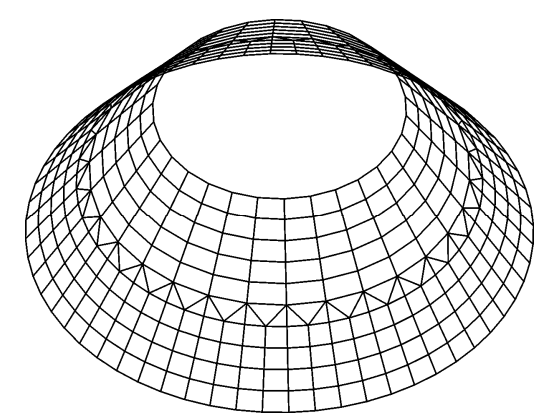

(c) Rectangular Grids: Decrease

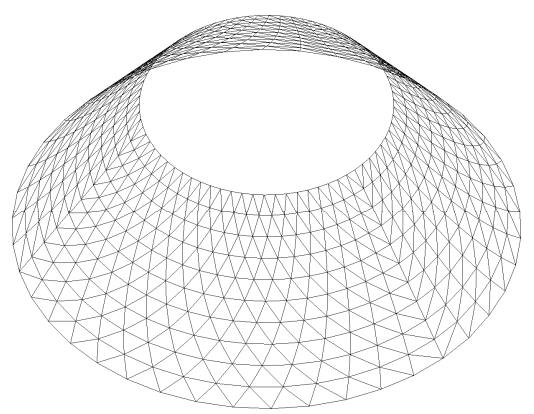

(d) Three-Dimensional Grids

Figure 12. Meshing Ways

\subsection{Evaluation Functions of Grid Quality}

The perfect space grid structures require that their grids are regular and the lengths of elements are uniform. Therefore, the evaluation functions of grid quality comprise statistical functions of grid shape and element length as follows:

(1) The sample of grid shape is quality coefficient $\left(G_{1}, G_{2}, \ldots, G_{m}\right)$, and the mean value is

$\bar{G}=\frac{1}{m} \sum_{i=0}^{m} G_{i}$.

The variance is

$S_{G}^{2}=\frac{1}{m-1} \sum_{i=0}^{m}\left(G_{i}-\bar{G}\right)^{2}$.

(2) The sample of the element length is $\left(L_{1}, L_{2}, \ldots, L_{n}\right)$, and the mean value is

$\bar{L}=\frac{1}{n} \sum_{i=0}^{n} L_{i}$. 
The variance is

$S_{L}^{2}=\frac{1}{n-1} \sum_{i=0}^{n}\left(L_{i}-\bar{L}\right)^{2}$

According to statistical theory, these evaluation functions represent the condition of the grid quality. For example, the bigger the mean value of quality coefficient expresses the better the grid shape, and the smaller variance means less difference of grid shape.

\section{DESIGN EXAMPLES}

There are several design examples to illustrate the above modeling technique for bionic space grid structure as follows:

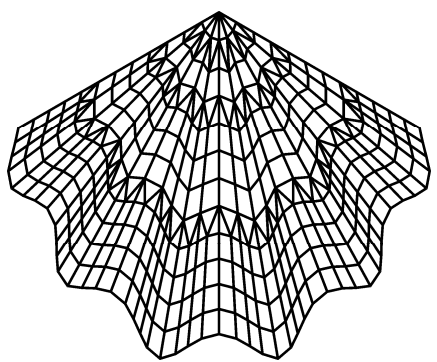

(a) Elevation View

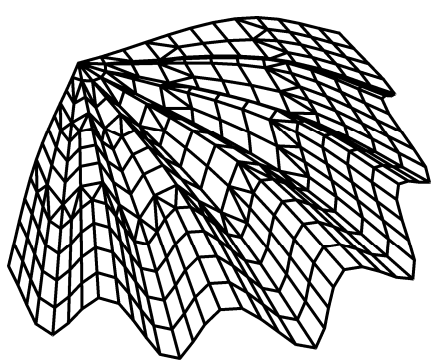

(b) Perspective View

\section{Figure 13. Scallop-Shaped Space Grid Structure}

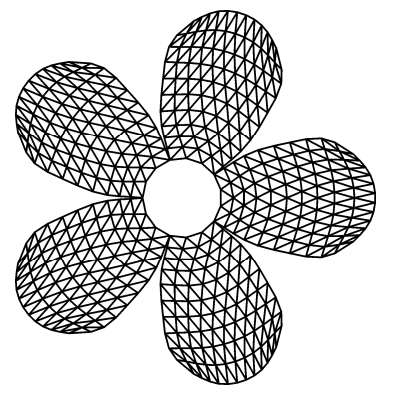

(a) Elevation View

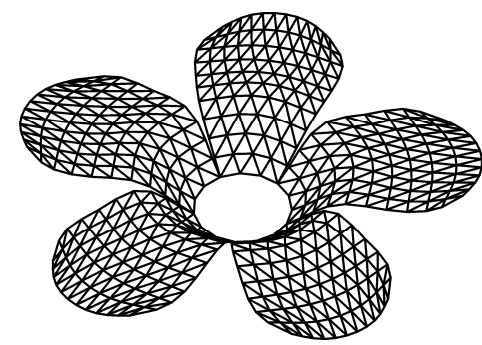

(b) Perspective View Figure 14. Pilosa-Shaped Space Grid Structure

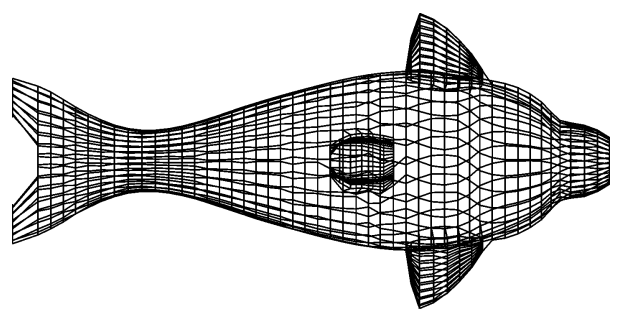

(a) Elevation View

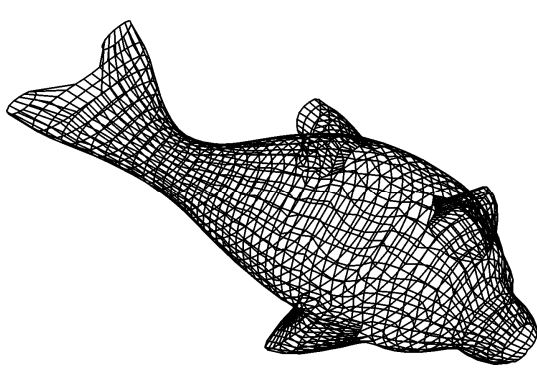

(b) Perspective View

Figure 15. Dolphin-Shaped Space Grid Structure 
(1) Scallop-shaped space grid structure (Figure 13)

- Rectangular grids: set grid number.

- Rational decrease on the top.

- The variance of element length: 0.250 .

(2) Pilosa-shaped space grid structure (Figure 14)

- Three-dimensional grids: set the number of central round and radial grids.

- The mean value of quality coefficient: 0.784 .

- The variance of quality coefficient: 0.022 .

- The variance of element length: 0.239 .

(3) Dolphin-shaped space grid structure (Figure 15)

- Rectangular grids: set grid number.

- The mean value of quality coefficient: 0.864 .

- The variance of quality coefficient: 0.011 .

\section{CONCLUSION}

When utilizing the modeling technique for bionic space grid structures, there are some conclusions that may be noticed:

(1) The acquisition method of data points derived from either bionics or reverse engineering is effective and convenient to imitate the natural patterns. Especially for complex surface, 3D measurement can be used to get sufficient data points. This is a really new idea to rebuild the bionic structure.

(2) The Imageware has powerful function to reconstruct the surface from data points, but it can not get the practical space grid structures directly. So the most important process of the modeling technique is the re-interpolation reflection meshing method.

(3) The re-interpolation reflection meshing method overcomes the inherent flaw of reflection meshing method. Though the surface obtained from the re-interpolation is not exactly the same as the initial interpolation, with sufficient new data points the output of re-interpolation can express the original surface in enough precision.

(4) The meshing ways and evaluation functions of grid quality adopted in this paper are necessary in the practice.

The bionic space grid structures display vogue and natural beauty, and have great potential application in the future. This rapid modeling technique will be helpful to accelerate the development of the bionic space grid structure.

\section{ACKNOWLEDGEMENT}

This study is funded by the Key Program of National Natural Science Foundation of China (No.50638050), the Doctoral Fund (No.20050335097) and the New Century Educational Talents Plan of Chinese Education Ministry (NCET-06-0517). This financial support is gratefully acknowledged. 


\section{REFERENCES}

[1] Harald, K., "Structural Design of Contemporary Free-form-architecture", New Olympics New Shell and Spatial Structures, IASS-APCS 2006 Symposium, Beijing, China, 2006.

[2] Stach, E., "Form-optimizing in Biological Structures: the Morphology of Seashells", Shell and Spatial Structures from Models to Realization, IASS 2004 Symposium, Montpellier, France, 2004.

[3] Raup, D.M., "Computer as Aid in Describing Form in Gastropod Shells", Science, 1962, Vol. 138, pp. 150-152.

[4] Várady, T. Martin, R.R. and Cox, J., "Reverse Engineering of Geometric Models-An Introduction", Computer-Aided Design, 1997, Vol. 29, No. 4, pp. 255-268.

[5] Barber, D. Mills, J. and Bryan, P., "Laser Scanning and Photogrammetry: 21st Century Metrology", CIPA Symposium, Potsdam, Germany, 2001.

[6] Balz, M. and Böhm, J., "Generating Shell Models and Their Realization by Photogrammetric Measurement", Shell and Spatial Structures from Models to Realization, IASS 2004 Symposium, Montpellier, France, 2004.

[7] Gong, X.Y., "Research on Generating Free Space Surface and Mesh Technology", Master Degree Dissertation, College of Civil Engineering and Architecture, Zhejiang University, Hangzhou, China, 2004. (in Chinese)

[8] Zhang, H., "Algorithms of Surface Modeling in Spatial Structure and their Program Realization", Master Degree Dissertation, College of Civil Engineering and Architecture, Zhejiang University, Hangzhou, China, 2005. (in Chinese)

[9] Cen P.C., "Parametric Description and Meshing Algorithm for the Surface of Spatial Structure", Master Degree Dissertation, College of Civil Engineering and Architecture, Zhejiang University, Hangzhou, China, 2006. (in Chinese)

[10] Li, N. and Luo, Y.Z., "Modeling Method for Bionic Latticed Shell", International Conference on Steel and Composite Structures, Manchester, 2007, pp.1005-1010.

[11] Luca, L.D. Veron, P. and Florenzano, M., "Reverse Engineering of Architectural Buildings Based on a Hybrid Modeling Approach", Computers \& Graphics, 2006, Vol. 30, pp.160-176.

[12] Farin, G., "Curves and Surfaces for Computer Aided Geometric Design: A Practical Guide", Academic Press, New York, 1993.

[13] DeBoor, C., "A Practical Guide to Splines”, Springer, New York, 1978.

[14] Zheng, Z.Z. Li, S.J. and Li, Z.G., "Classification and Comparison of Algorithm for Surface Mesh Generation”, Computer Aided Engineering, 1998, Vol. 1, pp. 53-58. (in Chinese) 